\title{
Enhanced Secured Communication Optimization in Data Packets Using Proxy Protocols
}

\author{
Shahul hameed $A^{a}$, Dr. A. Shaji george ${ }^{b}$, Bashiru aremu $^{c}$, Digvijay pandey ${ }^{d}$
}

Ph.D. Research Scholar, Department of Information and Communication Technology, Crown University, Int'l. Chartered Inc. (CUICI) Argentina Campus, South America ${ }^{\text {a }}$

Professor, Department of Information and Communication Technology, Crown University, Int'l. Chartered Inc. (CUICI) Argentina Campus, South America ${ }^{\mathrm{b}}$

Vice Chancellor, Crown University, Int'l. Chartered Inc. (CUICI) Argentina Campus, South America ${ }^{\mathrm{C}}$ Lecturer, Department of Technical Education (Government of U.P), India ${ }^{\mathrm{d}}$

E-mail: ashahul@yahoo.com ${ }^{\mathrm{a}}$; drashajigeorge@gmail.com ${ }^{\mathrm{b}}$, vc@ crownintl.education ${ }^{\mathrm{c}}$, digit11011989@gmail.com ${ }^{\mathrm{d}}$

\begin{abstract}
The principle objective of this paper is to build up a Computational Private Information Retrieval conventions on cloud design utilizing deletion code for made sure about information sending. These conventions are too costly in view of the fact that they are combining complicated numerical operations for the whole database. Essentially distributed storage engineering would provide a range of higher performing capacity staff that provides long-term internet storage management and even the distributed storage architecture. Putting information away and retrieving it in an external cloud system and public review plan creates difficulties and disputes about classification of information during transfers. On any outsider's case, this dispute is usually a massive amount of knowledge stored with the cloud worker. Also you can defeat this problem by means of various methods such as encryption, key encryption, etc. In either case, overall encryption plans maintain confidentiality of information during the exchange, thus restricting the utility of the capability system in parallel with this cycle. This is because a few activities have only been supported by scrambled knowledge. These techniques will cause disappointment. To building a protected stockpiling framework that underpins different capacities is testing when the capacity framework is appropriated and has no focal power.
\end{abstract}

Keywords: Cryptography, Secure storage system, Cloud storage architecture, framework, TPA, Proxy reencryption

\section{Introduction}

A distributed storage architecture consisting of a variety of capabilities provides long-term storage facilities across the Internet. The insertion of data into a cloud infrastructure of third parties raises fair data privacy issues [1]. Since certain activities are provided with encoded content, general encoding plans guarantee the classification of information thus restricting the use of the capacity network [2-4]. Without loading local computer storage and management, clients may use Cloud Storage for centralizing data saving and benefit from on-demand excellent tools and administering in a shared pool of configurable registration properties. However, given that customers currently do not have actual reassessed data power, Cloud Computing data integrity assurance is a difficult challenge, particularly for customers with substantial processing capacity [5-8].

Moreover, customers should be able to use shared storage as if local, without thinking about the need for their integrity to be validated. In this way, it is important to allow the public review 
capabilities for distributed storage in order to enable clients to rely on an external auditor (TPAThird Party Auditor). The evaluation loop must not introduce new vulnerabilities to the protection of customer information in order to securely present a solid TPA (Third Party Auditor), and must not familiarize customers with additional online weight [9-12]. In this article we propose a safe distributed storage device that facilitates public safety review. We further increase our results and make it easy and simultaneous for TPA to perform feedback for various customers. A rigorous safety and execution analysis shows that the existing plans are both credible and highly efficient. The skills workers will be unique and they are spread across many systems in order to make it possible to navigate information. It just contains encoded information from the information owners.

\section{Literature survey}

When the information sources are shared, we consider the question of creating a capability removal code over an organization. We assume that $\mathrm{n}$ storage hubs with limited memory and $\quad \mathrm{k}<\mathrm{n}$ sources can generate the information. In order to find out about $\mathrm{k}$ stock hubs and have the possibility to retrieve information, we need an information agency, which can present anywhere in the company. We are presenting Decentralized Erasure Codes which are straight codes with a specific random structure animated by arbitrary bipartisan network coding [4]. We demonstrate the perfect insufficiency of the decentralized eradication codes and decrease mail, storage and computation costs in relation to arbitrary straight coding. In this letter, we answer the question of transmitted arranged capability as there are various circulated sources which generate information which should be placed in separate warehouses efficient, each with limited memories [13-15].

Plautus is a cryptographic storage system which allows safe sharing of documents without placing a great deal of confidence in record staff. In order to secure and exchange data, it uses specifically cryptographic natives. Plautus provides an extremely adaptable key management system which allows individual customers to have direct authority on who gets admission to their documents. In Plautus we clarifying systems to reduce the number of encrypted keys exchanged between customers by means of record collections, to identify document readings and to write entry, to efficiently manage customer disclaimers and to enable an untrusted worker to authorize the composition of documents [16]. We also put together an Open AFS model of Plautus. Estimates of that model show that Plautus is virtually equal in terms of protection with overhead to frameworks which encrypt all traffic within the enterprise. As capacity frames and individual storage devices are arranged, they all must protect themselves from standard attacks on communications that are navigated by an untrustworthy, potentially accessible, network just as they attack the information they contain. This test is based on the fact that centralized stockpiling plays a fundamental role in enabling the easy exchange of knowledge often with protection of information.

Accessibility is an extraordinarily desirable yet negligibly built ability system property. Although 
some frameworks have tools for improving usability - for example repeat and deception recovery the system supervisor is periodically responsible for how to better develop these elements [17]. Tragically, hardly anyone has the ability to cope properly with the compromises involved, let alone adapt those decisions to changing circumstances. All aspects taken into account, most frames are statically ordered and have an uncertain understanding of how configuration in general execution or accessibility would interfere.

\section{Module Description}

The system or Platform IaaS/PaaS is the public-cloud-based ecosystem we rent from Linux (IaaS) or Microsoft (PaaS) (PaaS). Both have the capacity to host internet. The SaaS stack runs on your own hardware, perhaps in a virtualized format, making it private. In this project, we are specialized in private cloud technology. We function in the cloud in this situation. Use a public or hybrid cloud environment, otherwise use a public or community cloud environment if strict security requirements are required. As a result, the web server is integrated into the output, and when hosting the software the environment is seen in real time. Finally, in a cloud environment SaaS can be used entirely as IaaS/PaaS. We appreciate the difficulty of generating an erasure code for storage over a network as data sources are distributed across the cloud server. We infer in particular that $\mathrm{n}$ storage nodes with minimum space and $\mathrm{kn}$ data sources are available. We want a data collector who is able to consult $\mathrm{k}$ storage nodes and gather data across the network.

We use the randomized linear codes, which are inspired by network coding in randomly encrypted two-part graphics. We prove the optimum scarcity of decentralized erasure codes, which results in less cloud access, storage and processing costs compared to a random linear code. It can be found on both physical and virtual network models with advanced encoding. This is more efficient on cloud networks. Cripting of proxies is an encryption method that permits third-party processors to alter a cipher text which is encrypted and decrypted to another party for one entity. Standard system of symmetrical or asymmetrical encoding are similar to proxy encoding systems. You will create a reencryption key for the private key and the delegated user key. The recipient is a key holder. As the input for the re-encryption feature, the proxy uses the re-encryption key to translate cipher text into the user's allocated key. The asymmetric proxy re-encryption systems are bi-directional and unidirectional. The proxy encryption schemes allows a cipher text to be encrypted again an unlimited number of times. Proxy re-encryption should not be confused with proxy signatures that are a separate form of structure for another purpose.

\section{Data flow}

Asp.net is a software application framework designed to have interactive website pages from the side of the worker. Microsoft developed it to allow software engineers to build dynamic pages, web 
applications and project management. It was first delivered in January 2002 with variant 1.0 of the.net system, and is the replacement to Microsoft's dynamic worker pages (asp) innovation. Asp.net is built on the normal runtime (clr) of the language that allows software engineers to create asp.net code using any upheld.net language. The asp.net cleanser increase scheme enables the cleanser messages of asp.net segments to be managed.

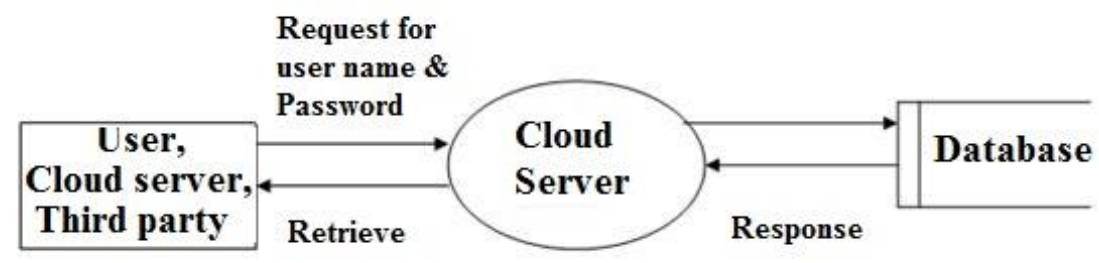

Fig.1.Data flow

Asp.net 1.0 was delivered as part of the 1.0 adaptation on January 5, 2002, after four years of improvements and an increase of trial deliveries in 2000 and 2001. On January 5, 2002, Indeed, several books were exposed on the website as well as Microsoft's extensive advancement as part of its Network administration base even before the distribution. Scott Guthrie is a supervisor of item units for asp.net, and was upgraded quickly and Type 1.1 was submitted as a Windows Worker on 24 April 2003.

The document platform conveyed to the cloud is a record framework that allows many customers to approach related information/document with major activities (make, erase, change, read, compose). Each text can be divided into several parts known as lumps. In faraway machines each lump is packed away. In the documentation in a progressive tree where the hubs talk to the indexes, information is regularly provided. It then promotes the fair performance of uses. Any approaches to exchange records in a disseminated engineering are different. Each structure should be reasonable for a particular purpose based on the complexity or simplicity of the application. 


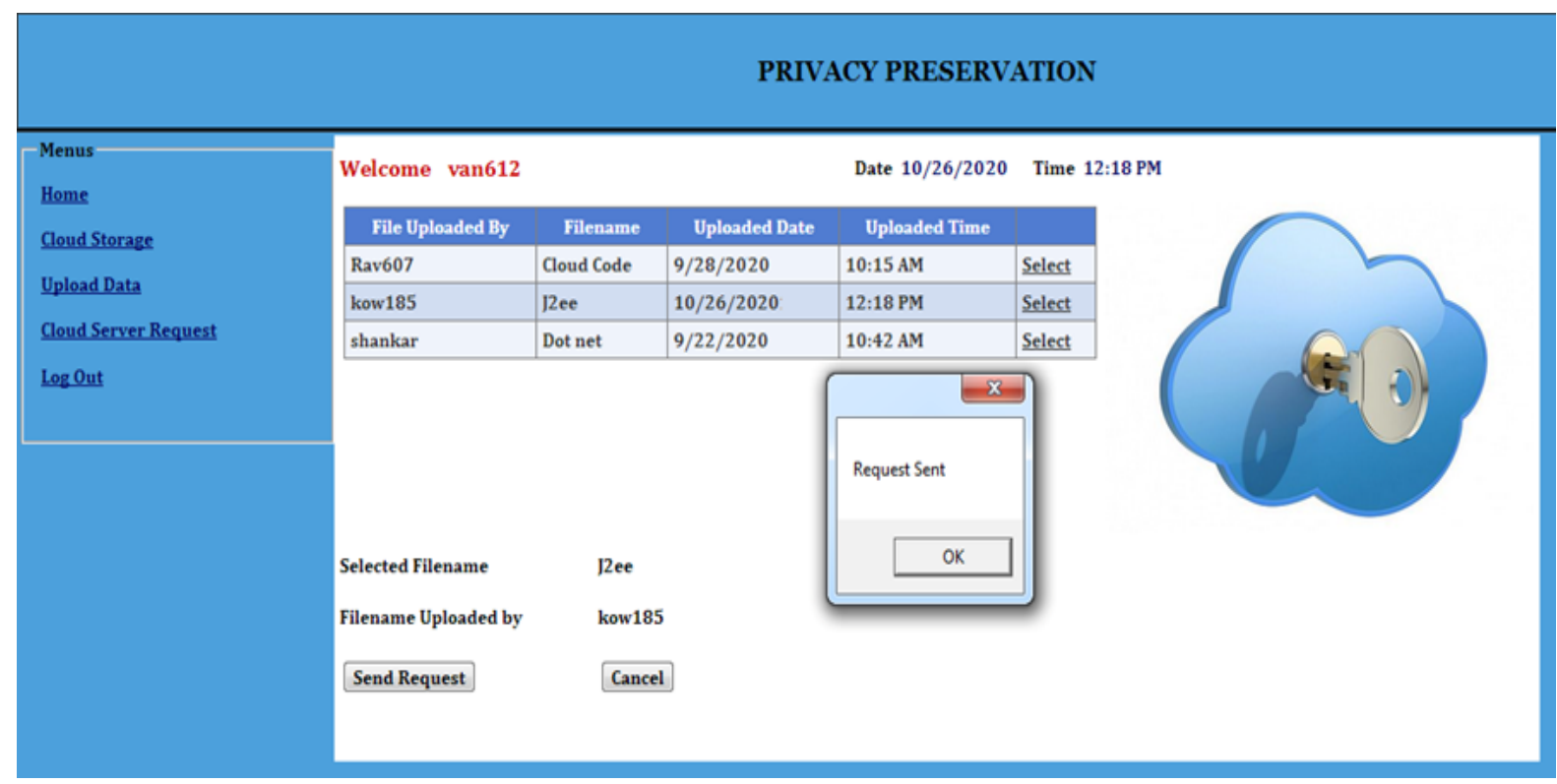

Fig.2.System process

\section{System Analysis}

Two of the major issues regarding distributed storage are uncompromising quality and security. Customers are reluctantly confident that they will access their data at any moment they need to and so none other organization can. Once they are sure they can have access to their data. Most implementations use a variety of identity management techniques including authentication systems that require a client's name and a secret key to be created. Procedure for approval - the customer tracks who accessed data in the cloud environment has been granted.

For different partnerships there are different degrees of clearance. A front-line staff, for example, may only have selective access to records stored in a cloud platform and a large amount of access to information from the head of HR. Many people still think that the data stored on a remote storage facility is unprotected with these protections. A programmer can often find a secondary automatic passage and get access to details. Programmers may also try to take control over the actual data-saving machines. A frustrated delegate may modify or delete material by using their checked client name and secret speech. 


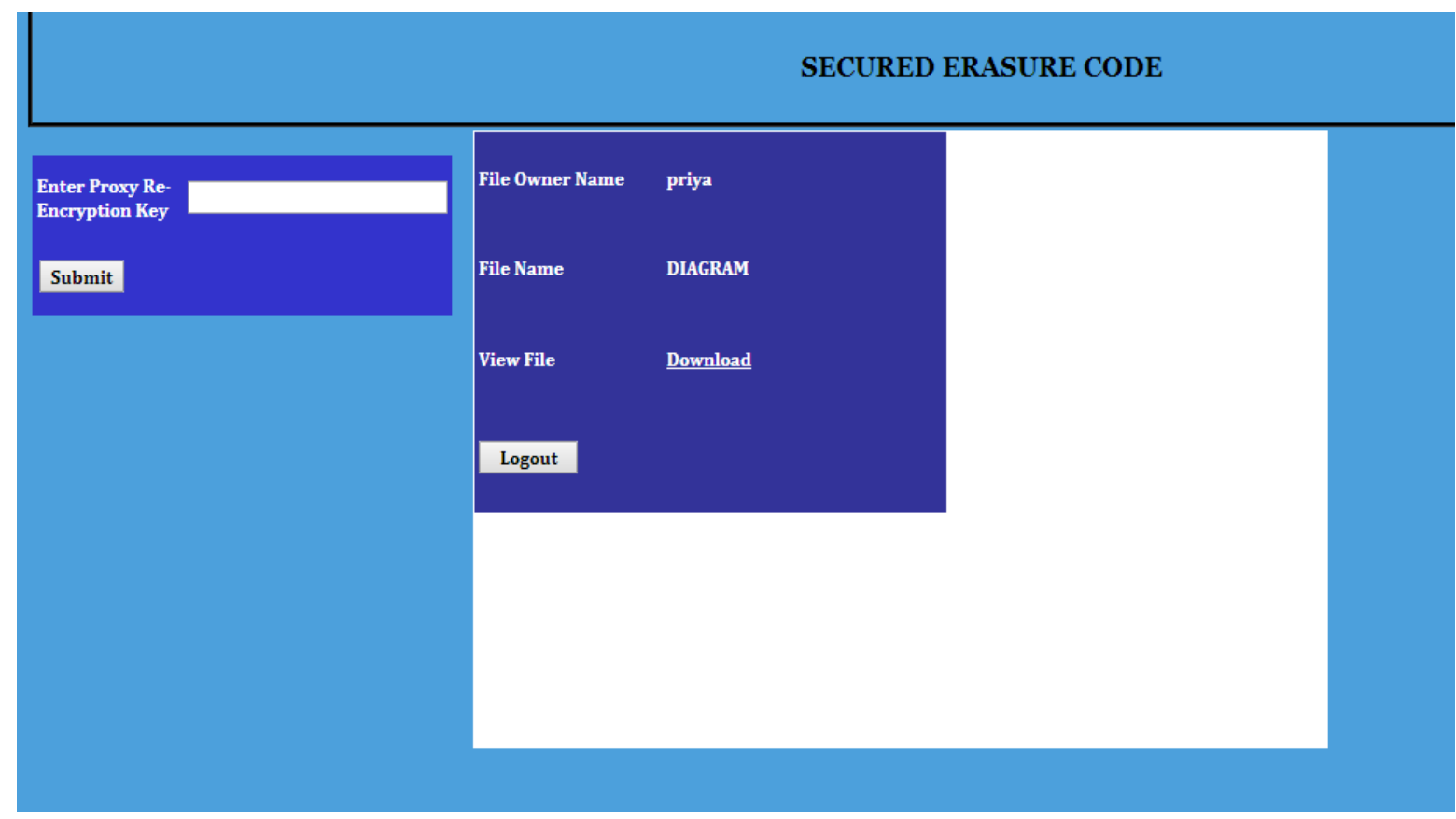

Fig.3.Time based encrypting

Distributed storage providers spend a great deal of resources into safety mechanisms to reduce the possibility of computer robbery or defamation. The other big concern, trustworthiness and security is just as critical. An unreliable distributed storage infrastructure is a weakness. Nobody wants to store data on a faulty device and nobody wishes to trust a financially unstable organization. Cloud providers' reputations are all like this. It is in each organization's best interests to have the safest and most reliable operation. If a company is not able to fulfill these specific consumer expectations, it will not have the chance on the industry.

\section{Conclusion}

We thus conclude that all the results have been generated by the logical submission. This paper includes capacity and key workers in a distributed storage system. We coordinate intermediate reencryption and eradication codes over varieties recently proposed. The intermediate recryption of limits supports the dissemination, transfer and incompletion of unscrewing activities. Any main worker needs to unscramble the two code word pictures in our system to decipher a message from $\mathrm{k}$ squares that are scraped and encoded. Using intermediate re-encryption limits, we present a stable distributed storage system which provides secure stockpiling of information and secure information in a decentralized structure. Any capability worker often autonomously codes and re-encrypts and openly scrambles fractional jobs. Our capacity architecture and certain content models that can be addressed recently are extremely viable. Our capabilities are designated as centers of capability in a capacity system for the disposal of addressable squares for substances. As control hubs, our main employees use a front-end layer such as a traditional record frame interface. Further research on definite 
International Journal of Intelligent Communication, Computing and Networks

Open Access Journal (ISSN: 2582-7707)

https://doi.org/10.51735/ijiccn/001/15

participation is essential.

\section{References}

1. Scott-Hayward, S., \& Arumugam,T. (2018)“'OFMTL-SEC: State-based security for software de_ned networks," in Proc. IEEE Conf. Netw. Function Virtualization Softw. De_ned Netw. (NFV-SDN), pp. 1-7.

2. Dargahi, T., Caponi, A., Ambrosin, M., Bianchi, G., \& Conti, M.( 2017)“'A survey on the security of stateful SDN data planes," IEEE Commun. Surveys Tuts., vol. 19, no. 3, pp. 1701-1725.

3. Ai, J., Guo, Z., Chen, H., \& Cheng, G.( 2019)"Improving the routing security in software-de_ned networks," IEEE Commun. Lett., vol. 23, no. 5, pp. 838-841.

4. Mattos, D. M. F., \& Duarte, O. C. M. B. (2016)“'AuthFlow: Authentication and access control mechanism for software de_ned networking," Ann. Telecommun., vol. 71, nos. 11_12, pp. 607-615.

5. Wang, T., Guo, Z., Chen, H., \& Liu, W.( 2018) "BWManager: Mitigating denial of service attacks in software-de_ned networks through bandwidth prediction," IEEE Trans. Netw. Service Manage., vol. 15, no. 4, pp. $1235-1248$

6. Sunghwan kim, Seunghyun yoon, Jargalsaikhan narantuya , \& Hyuk lim (2020) , "Secure Collecting, Optimizing, and Deploying of Firewall Rules in Software-Defined Networks "IEEE Access, pp. 15166-15177.

7. Bhagwan, R., Tati, K., Cheng, Y.C., Savage, S., \& Voelker ,G.M.( 2004) "Total Recall: System Support for Automated Availability Management,"Proc. First Symp. Networked Systems Design and Implementation (NSDI),pp. 337-350

8. Dimakis, A.G., Prabhakaran, V.,\& Ramchandran, K.( 2005) "Ubiqui-tous Access to Distributed Data in Large-Scale Sensor Net-works through Decentralized Erasure Codes,'Proc. Fourth Int'l Symp. Information Processing in Sensor Networks (IPSN),pp. 111-117.

9. Dimakis, A.G., Prabhakaran, V., \& Ramchandran K.( 2006) "Decen-tralized Erasure Codes for Distributed Networked Storage,"IEEE Trans. Information Theory,vol. 52, no. 6 pp. 2809-2816.

10. Mambo M., \& Okamoto, E.( 1997) "Proxy Cryptosystems: Delegation of the Power to Decrypt Ciphertexts," IEICE Trans. Fundamentals of Electronics, Comm. and Computer Sciences,vol. E80-A, no. 1, pp. 54-63.

11. Blaze, M., Bleumer, G., \& M. Strauss. ( 1998) “Divertible Protocols and Atomic Proxy Cryptography,”Proc. Int'l Conf. Theory and Applica-tion of Cryptographic Techniques (EUROCRYPT),pp. 127-144.

12. Ateniese, G., Fu, K., Green M., \& S. Hohenberger. ( 2006) "Improved Proxy Re-Encryption Schemes with Applications to Secure Distributed Storage,"ACM Trans. Information and System Security, vol. 9, no. 1, pp. 130.

13. Tang, Q. ( 2008) "Type-Based Proxy Re-Encryption and Its Construction," Proc. Ninth Int'l Conf. Cryptology in India: Progress in Cryptology (INDOCRYPT),pp. 130-144.

14. Ateniese, G., Benson, K., \& Hohenberger S. ( 2009) "Key-Private Proxy Re-Encryption, "Proc. Topics in Cryptology (CT-RSA),pp. 279-294.

15. Shao J., \& Cao, Z.( 2009) “CCA-Secure Proxy Re-Encryption without Pairings,"Proc. 12th Int'l Conf. Practice and Theory in Public Key Cryptography (PKC),pp. 357-376.

16. Ateniese, G., Burns, R., Curtmola, R., Herring, J., Kissner, L., Peterson, Z.,\& Song, D.

"Provable Data Possession at Untrusted Stores,"Proc. 14th ACM Conf. Computer and Comm. Security (CCS), pp. 598-609. 
International Journal of Intelligent Communication, Computing and Networks

Open Access Journal (ISSN: 2582-7707)

https://doi.org/10.51735/ijiccn/001/15

17. Ateniese, G., Pietro, R.D., Mancini, L.V., \& Tsudik, G.( 2008) "Scalable and Efficient Provable Data Possession,"Proc. Fourth Int'l Conf. Security and Privacy in Comm. Netowrks (SecureComm),pp. 1-10. 